(2) Open Access Full Text Article

REVIEW

\title{
Amrubicin: potential in combination with cisplatin or carboplatin to treat small-cell lung cancer
}

This article was published in the following Dove Press journal:

Drug Design, Development and Therapy

31 July 2013

Number of times this article has been viewed

\author{
Qian Ding \\ Jinbiao Zhan \\ Department of Biochemistry, \\ Zhejiang University School \\ of Medicine, Hangzhou, \\ People's Republic of China
}

Correspondence: Jinbiao Zhan

Department of Biochemistry,

Zhejiang University School of Medicine,

866 Yuhangtang Rd, Hangzhou 310058,

People's Republic of China

Tel $+8657 \mid 88208272$

Fax +86 57। 88208273

Email jzhan2k@zju.edu.cn
Abstract: Small-cell lung cancer (SCLC) is the most aggressive form of lung cancer characterized by early metastasis and high mortality. In recent years, monotherapy and combination therapy of amrubicin with cisplatin or carboplatin has been actively studied and shown promise for the treatment of extensive disease SCLC (ED-SCLC). In this article, we summarize clinical trials of both monotherapy and combination therapy with amrubicin conducted in Japan, the USA, and the European Union. The results suggest that the clinical outcome of amrubicin therapy may be associated with genetic variations in patients. Further study of combination regimens in patients of different ethnicities is warranted.

Keywords: amrubicin, cisplatin, carboplatin, combination therapy, small-cell lung cancer, antibody-drug conjugates

\section{Introduction}

Lung cancer is the most common cancer and causes 1.38 million deaths annually worldwide. ${ }^{1}$ The major types of lung cancer are small-cell lung carcinoma (SCLC) and non-small-cell lung carcinoma (NSCLC). SCLC comprises approximately $15 \%$ of all lung cancers and over 25,000 people are diagnosed with SCLC in the US each year. ${ }^{2}$ Traditionally, SCLC is classified into "limited disease" (LD) or "extensive disease" (ED), depending on the degree of spread. ${ }^{3}$ This type of lung cancer is highly aggressive and approximately two-thirds of SCLC patients have disease spread at diagnosis.

In the past few decades, SCLC has mainly been treated with combinations of a platinum agent (cisplatin or carboplatin) with etoposide or irinotecan. ${ }^{4}$ The combination regimen has long been the standard chemotherapy; however, only little improvement in survival has been achieved over the last 30 years, reflecting a need to develop more effective therapies. ${ }^{5,6}$ In recent years, amrubicin alone and in combination with other chemotherapeutic agents, such as cisplatin and carboplatin, have been actively studied in Japan and have shown promise. ${ }^{7,8}$ Now more clinical studies have been conducted to evaluate the efficacy and safety of amrubicin in Western populations. ${ }^{9}$ This review summarizes the clinical studies of amrubicin in combination with cisplatin or carboplatin for the treatment of ED-SCLC, aiming for further improvement of the combination regimens.

\section{Chemistry and mechanisms of action}

Amrubicin (brand name Calsed ${ }^{\circledR}$, Sumitomo Pharmaceuticals, Japan) is a synthetic anthracycline used for treatment of lung cancer including SCLC and NSCLC. It was developed by Sumitomo Pharmaceuticals and first approved in Japan in 2002. 
Amrubicin has a structure similar to doxorubicin and works as a potent inhibitor of topoisomerase II. ${ }^{10}$ Preclinical studies show that amrubicin has better antitumor activity than doxorubicin in several xenograft models of human cancers and causes almost no cardiotoxicity. ${ }^{11,12}$ The lower cardiotoxicity of amrubicin results from lower levels of accumulation and metabolic advantages over doxorubicin. ${ }^{13,14}$

It has been demonstrated that amrubicin itself has a weak antitumor effect and converts to the active form amrubicinol by reduction of the $\mathrm{C}$-13 ketone group to a hydroxy group in vivo (Figure 1). Amrubicinol has about 10- to 100-fold greater anticancer activity than the prodrug amrubicin. ${ }^{15}$

The mechanism of action of anthracyclines has been extensively investigated. Several lines of evidence indicate that they interact with many macromolecules in the cell. Amrubicin is a potent inhibitor of topoisomerase II, which relaxes supercoils of deoxyribonucleic acid (DNA) for replication. Through facilitating the formation of a cleavable topoisomerase II-DNA complex, amrubicin prevents the double-helix of DNA from being resealed and thereby arrests DNA synthesis in rapidly-growing cancer cells. ${ }^{10}$ In addition, as a member of the anthracyclines, the aromatic chromophore moiety of amrubicin intercalates between adjacent base-pairs of DNA, while the six-membered sugar interacts with flanking base-pairs near the intercalation site in the minor groove, resulting in the breakage of singleand double-strand DNA. ${ }^{16-18}$ Eventually, the anthracycline induces apoptosis in different cancer cells through several signaling pathways involved in nuclear factor kappa-B $(\mathrm{NF}-\kappa \mathrm{B})$ activation, p53-dependence, and caspase-3 activation. $^{19}$

\section{Pharmacokinetics and pharmacodynamics}

Several pharmacokinetic studies of amrubicin and its active metabolite amrubicinol have been reported. ${ }^{20-22}$ When amrubicin was administered at a dose of $45 \mathrm{mg} / \mathrm{m}^{2} /$ day in a bolus injection for 3 consecutive days, the area under the curve concentration-time (AUC) for amrubicin was $13.490 \mu \mathrm{g}$ hour $/ \mathrm{mL}$ and for amrubicinol was $2.585 \mu \mathrm{g}$ hour $/ \mathrm{mL} .{ }^{18}$ The apparent total clearance of amrubicin was $15.4 \mathrm{~L} /$ hour with large inter-individual variability (coefficient of variation $=49.8 \%$ ). Another study showed that the plasma concentration peaked immediately after a bolus intravenous injection of amrubicin and declined rapidly in a biexponential manner thereafter, whereas the level of amrubicinol decreased more slowly. The apparent total clearance of amrubicin has a large inter-individual variability, despite adjustment of dosage for body surface area. ${ }^{21}$ These studies suggest that monitoring plasma concentrations of amrubicin and amrubicinol may be necessary to obtain the optimal dosage for each patient. ${ }^{20,21}$

A relationship between the plasma concentration of the metabolite amrubicinol and hematological toxicity has been reported..$^{22}$ In 35 patients who received 30 or $40 \mathrm{mg} / \mathrm{m}^{2} /$ day for 3 consecutive days, a significant relationship between amrubicinol concentration on day 4 and the level of toxicity (neutropenia, leukopenia, and anemia) was found. ${ }^{22}$ Recently, a pharmacokinetic (PK) and pharmacodynamic (PD) study of amrubicin and amrubicinol in patients with lung cancer was conducted. ${ }^{23}$ Amrubicin $\left(35-40 \mathrm{mg} / \mathrm{m}^{2}\right.$ ) was administered to 21 lung-cancer patients for 3 consecutive days every 3-4 weeks. The concentrations of amrubicin and amrubicinol in plasma were quantified with high-performance liquid chromatography (HPLC). The overall PK profile of amrubicin obeys a three-compartment model, while that of amrubicinol follows a one-compartment model with a firstorder metabolic process. The PK-PD analysis indicated that the higher maximal plasma concentration $\left(\mathrm{C}_{\max }\right)$ and $\mathrm{AUC}$ of amrubicinol is directly correlated with major toxicity (neutropenia). ${ }^{24}$ In the case of combination with cisplatin, an in vitro study showed that cisplatin does not influence the metabolism of amrubicin and amrubicinol in either human liver microsomes or the cytosol. ${ }^{25}$ The metabolic pathway of amrubicin includes the reduction of the carbonyl group to a hydroxyl group at the C-13 site by carbonyl reductase, and inactivation by nicotinamide adenine dinucleotide phosphate (NADPH):quinone oxidoreductase (NQO) and
A

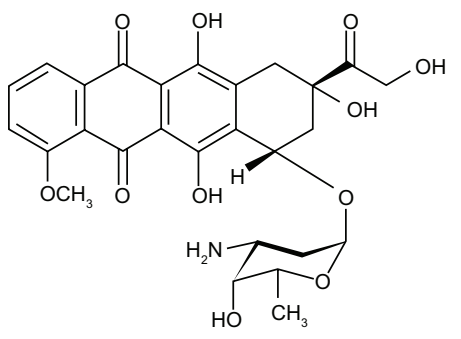

B

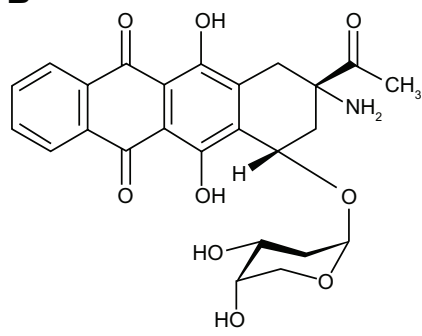

C

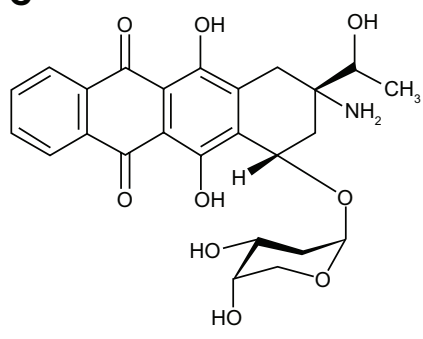

Figure I Chemical structures of doxorubicin (A), amrubicin (B), and amrubicinol (C). 
NADPH-P450 reductase. ${ }^{25}$ The quinones are reduced to hydroquinones in a single two-electron step. Recently, it was reported that $\mathrm{C609T}$ polymorphism of NADPH quinone oxidoreductase 1 (NQO1) in patients with lung cancer is correlated with amrubicinol levels in plasma and hematological toxicity of amrubicin. ${ }^{26} \mathrm{~A}$ single nucleotide polymorphism in C609T of NQO1 enhanced the susceptibility of cancer cells to amrubicin, which may be used as a predictive biomarker for clinical response. ${ }^{27}$

\section{Clinical studies Phase I trials}

From 2005 to 2012, several Phase I trials have been reported for amrubicin alone and in combination with cisplatin or carboplatin for the treatment of lung cancer and SCLC (Table 1). ${ }^{20,28-31}$ Phase I studies are designed to determine the maximum-tolerated doses (MTDs) and the recommended doses (RDs) for Phase II studies. Amrubicin was administered daily by intravenous injection (IV) for 3 consecutive days every 3-4 weeks. For monotherapy with amrubicin, the MTD was $40 \mathrm{mg} / \mathrm{m}^{2}$ and the RD was $35 \mathrm{mg} / \mathrm{m}^{2}$ while in the presence of cisplatin $\left(60 \mathrm{mg} / \mathrm{m}^{2}\right)$, the MTD increased to $45 \mathrm{mg} / \mathrm{m}^{2}$ and the $\mathrm{RD}$ to $40 \mathrm{mg} / \mathrm{m}^{2} \cdot{ }^{20,29}$ Two studies of combination therapy with carboplatin have been conducted. The MTD and RD of amrubicin were the same as in monotherapy, while the dose of carboplatin in elderly patients was reduced from a fixed target AUC of five to four. ${ }^{31,32}$ The common toxicities, neutropenia, leukopenia, thrombocytopenia, febrile neutropenia, were observed in these trials. Recently, a Phase I trial of weekly administration of amrubicin was reported ${ }^{28}$ Sixteen patients (seven with SCLC and nine with NSCLC) participated and the MTD was $65 \mathrm{mg} / \mathrm{m}^{2}$ and the RD $60 \mathrm{mg} / \mathrm{m}^{2}$. The best response rate was $15.4 \%$, and adverse events with this regimen were tolerable, so the adjusted regimen demands further study. In order to reduce the adverse effects of amrubicin, granulocyte colony-stimulating factor (G-CSF) has been used recently to support the therapy. The prophylactic use of G-CSF allowed the dose of amrubicin to be raised $40 \%$ in the original regimen. ${ }^{32}$

\section{Phase II trials}

Several Phase II studies have been conducted in Japan, the USA, and the European Union (EU) for the treatment of SCLC. The results are summarized in Table 2. For monotherapy with amrubicin, five studies have been reported (three in Japan and two in the USA and EU). In the three studies in Japan, patients with refractory or relapsed SCLC were enrolled. ${ }^{33-35}$ The first group contained patients who experienced progression of disease occurring within 3 months of completion of initial therapy, including people who never responded and people who responded but relapsed quickly (refractory group), and the second group contained patients who relapsed more than 3 months from completion of initial therapy (sensitive group).

Amrubicin was administered at $35-40 \mathrm{mg} / \mathrm{m}^{2}$ IV for 3 consecutive days, every $3-4$ weeks. The overall response rate (ORR) in the sensitive group was $52 \%-60 \%$ and in the refractory group $17 \%-50 \%$. The median progression-free survivals (PFSs) in the refractory groups and the sensitive groups were 2.6-4.0 months and 3.9-4.5 months, respectively. The median survival times (MSTs) in the refractory groups were 5.3-11 months and in the sensitive groups were 9.2-12 months. The main toxicity was hematologic toxicities including neutropenia, thrombocytopenia, and anemia with febrile neutropenia, while nonhematologic toxicity was mild. These results indicated that amrubicin has significant efficacy against SCLC, with acceptable toxicity in Japanese patients. ${ }^{33-35} \mathrm{In}$ the second trial, the effects of amrubicin and topotecan were compared. ${ }^{34}$ Eligible patients with SCLC previously treated with platinum-based chemotherapy were randomly assigned to receive amrubicin $\left(40 \mathrm{mg} / \mathrm{m}^{2}\right.$, days 1 through 3$)$ or topotecan $\left(1.0 \mathrm{mg} / \mathrm{m}^{2}\right.$, days 1 through 5$)$. Of 60 patients enrolled, 59 (36 sensitive and 23 with refractory relapse) were assessable for efficacy and safety evaluation. Amrubicin treatment resulted in a significantly higher ORR than topotecan (38\% versus $13 \%)$. The median PFS was 3.5 months for amrubicin and 2.2 months for topotecan treatment. One treatment-related death due to infection occurred in the amrubicin group. The results indicated that amrubicin is superior to topotecan for the treatment of previously-treated SCLC patients.

In order to investigate the efficacy of amrubicin in Western patients, recently two Phase II trials of amrubicin as secondline therapy in SCLC patients with refractory or sensitive relapse have been completed in the USA and $\mathrm{EU}^{33-37}$ In the first trial, patients with refractory SCLC (either with progressive disease as the best response or progression within 90 days of first-line therapy) received amrubicin $\left(40 \mathrm{mg} / \mathrm{m}^{2} /\right.$ day for 3 days every 21 days). Seventy-five eligible patients were enrolled and 69 patients received an average of four cycles of treatment. The ORR was $21.3 \%$ with one complete response $(1.3 \%)$ and 15 partial responses (20\%). The median PFS was 3.2 months and the overall survival (OS) 6.0 months. The ORR in 43 patients who did not respond to first-line therapy was $16.3 \%$. Adverse events occurred as neutropenia (67\%), thrombocytopenia (41\%), and anemia (30\%), with febrile 


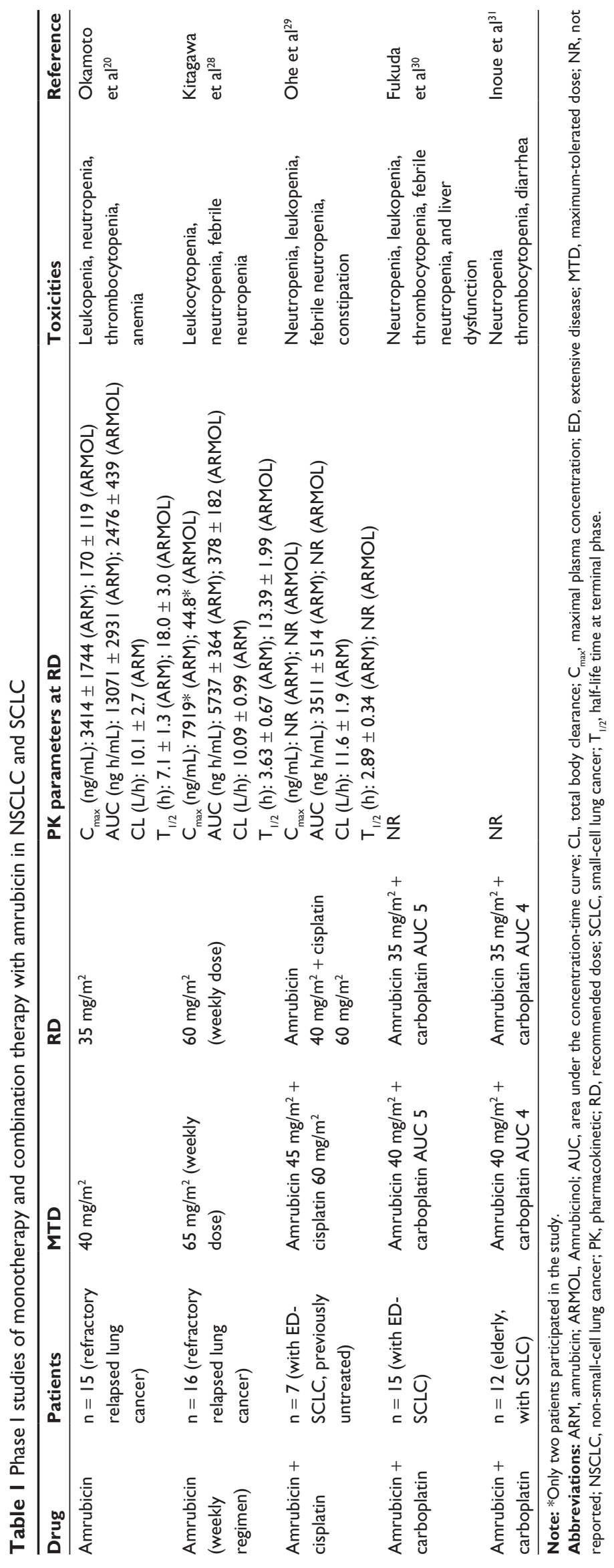


Table 2 Phase II studies of amrubicin monotherapy or combination therapy with a platinum agent in SCLC patients

\begin{tabular}{|c|c|c|c|c|c|c|c|}
\hline Drug & Patients & $\mathbf{n}$ & ORR (\%) & PFS (months) & MST (months) & Country & Reference \\
\hline \multicolumn{8}{|l|}{ Monotherapy } \\
\hline \multirow[t]{2}{*}{ Amrubicin } & Sensitive relapse & 44 & 52 & 4.2 & 11.6 & Japan & Onoda et $\mathrm{a}^{33}$ \\
\hline & Refractory relapse & 16 & 50 & 2.6 & 10.3 & & \\
\hline Amrubicin & Sensitive relapse & $17(19)$ & $53(2 I)$ & $3.9(3.0)$ & $9.9(11.7)$ & Japan & Inoue et $\mathrm{a}^{34}$ \\
\hline (comparator: topotecan) & Refractory relapse & $12(11)$ & $17(0)$ & $2.6(1.5)$ & $5.3(5.4)$ & & \\
\hline \multirow[t]{2}{*}{ Amrubicin } & Sensitive relapse & 10 & 60 & 4 & 12 & Japan & Kaira et $\mathrm{al}^{35}$ \\
\hline & Refractory relapse & 19 & 36.8 & 4 & II & & \\
\hline Amrubicin & Refractory relapse & 75 & 21.3 & 3.2 & 6 & $\begin{array}{l}\text { USA } \\
\text { and EU }\end{array}$ & Ettinger et $\mathrm{a}^{36}$ \\
\hline $\begin{array}{l}\text { Amrubicin } \\
\text { (comparator: topotecan) }\end{array}$ & Sensitive relapse & $\begin{array}{l}50 \\
(26)\end{array}$ & $\begin{array}{l}44 \\
(15)\end{array}$ & $\begin{array}{l}4.5 \\
(3.3)\end{array}$ & $\begin{array}{l}9.2 \\
(7.2)\end{array}$ & USA & Jotte et $\mathrm{a}^{37}$ \\
\hline \multicolumn{8}{|l|}{ Combination therapy } \\
\hline Amrubicin + cisplatin & $\begin{array}{l}\text { With ED-SCLC, } \\
\text { previously untreated }\end{array}$ & 41 & 87.8 & NA & 13.6 & Japan & Ohe et $\mathrm{al}^{29}$ \\
\hline Amrubicin + cisplatin & $\begin{array}{l}\text { With ED-SCLC, } \\
\text { previously untreated }\end{array}$ & 33 & 77 & 6.9 & 11.1 & EU & O'Brien et a ${ }^{38}$ \\
\hline \multicolumn{8}{|l|}{ Comparators } \\
\hline Amrubicin alone & & 33 & 61 & 5.2 & II.I & & \\
\hline Cisplatin etoposide & & 33 & 63 & 5.8 & 10 & & \\
\hline Amrubicin + carboplatin & $\begin{array}{l}\text { Chemotherapy-naïve } \\
\text { elderly patients with } \\
\text { SCLC }\end{array}$ & 36 & 89 & 5.8 & 18.6 & Japan & Inoue et $\mathrm{a}^{31}$ \\
\hline \multirow[t]{3}{*}{ Amrubicin + carboplatin } & $\begin{array}{l}\text { Previously treated } \\
\text { patients }\end{array}$ & & & & & Japan & Hirose et al ${ }^{39}$ \\
\hline & Sensitive relapse & 12 & 58.3 & 5 & 10 & & \\
\hline & Refractory relapse & 13 & 15.4 & 2 & 5 & & \\
\hline
\end{tabular}

Abbreviations: ED, extensive disease; EU, European Union; MST, median survival time; $n$, number; ORR, overall response rate; PFS, median progression-free survival; SCLC, small-cell lung cancer; NA, not applicable.

neutropenia in $12 \%$. No early cardiotoxicity was observed, even with cumulative amrubicin doses exceeding $750 \mathrm{mg} / \mathrm{m}^{2}$. These results demonstrated that monotherapy with amrubicin as second-line therapy shows promising activity and an acceptable safety in Western patients with refractory SCLC. ${ }^{36}$ The second randomized Phase II trial to compare single-agent amrubicin with topotecan as second-line treatment in patients with SCLC sensitive to first-line platinum-based chemotherapy was conducted in the USA. ${ }^{37}$ Seventy-six patients were enrolled in the study. Of them, 50 patients were randomly assigned to the amrubicin group and 26 to the topotecan group. Patients received amrubicin $\left(40 \mathrm{mg} / \mathrm{m}^{2} /\right.$ day in a 5 -minute IV infusion, days 1 through 3, every 21 days) or topotecan $\left(1.5 \mathrm{mg} / \mathrm{m}^{2} /\right.$ day in a 30 -minute IV infusion, days 1 through 5 , every 21 days). Amrubicin resulted in a significantly higher ORR than topotecan (44\% versus $15 \%$ ). The median PFS was 4.5 months for amrubicin and 3.3 months for topotecan, while the median OS was 9.2 months for amrubicin, and 7.6 months for topotecan. Worse adverse effects (neutropenia and thrombocytopenia) occurred in the topotecan group. ${ }^{37}$ These results demonstrated that amrubicin has better activity than topotecan as second-line treatment in Western patients with SCLC sensitive to first-line platinum-based chemotherapy.
Monotherapy with amrubicin has shown positive effects in patients with SCLC in previous studies. Since SCLC patients are traditionally sensitive to platinum agents, it would be interesting to evaluate the efficacy of combination therapy of amrubicin with cisplatin or carboplatin. Three Phase II trials of the combination therapy of amrubicin with platinum agents have been conducted in Japan. The first study in previously-untreated patients with ED-SCLC was reported in 2005. ${ }^{29}$ Forty-one patients received amrubicin $\left(40 \mathrm{mg} / \mathrm{m}^{2}\right.$, days $1-3)$ and cisplatin $\left(60 \mathrm{mg} / \mathrm{m}^{2}\right.$, day 1$)$, and $22(54 \%)$ patients completed four cycles without dose modification. However, ten (31\%) patients had a reduction in the amrubicin dose at the fourth cycle. The ORR was $87.8 \%$ and the MST was 13.6 months, while the 1 -year survival rate was $56.1 \%$. Adverse effects included grade 3/4 neutropenia and leukopenia occurred in $95.1 \%$ and $65.9 \%$ of patients, respectively. The combination therapy of amrubicin and cisplatin shows a significant efficacy in patients with previously-untreated ED-SCLC.

Recently, a European group reported a multicenter Phase II study of amrubicin as the single agent or in combination with cisplatin versus cisplatin etoposide as the first-line treatment in patients with ED-SCLC. ${ }^{38}$ From June 2006 to 
July 2009, 99 patients with previously-untreated ED-SCLC were randomized: 33 in each arm. Eligible patients received 3 weekly cycles of either amrubicin alone $\left(45 \mathrm{mg} / \mathrm{m}^{2} \mathrm{IV}\right.$ on days $1-3$; A), cisplatin $60 \mathrm{mg} / \mathrm{m}^{2} \mathrm{IV}$ on day 1 and amrubicin $40 \mathrm{mg} / \mathrm{m}^{2} \mathrm{IV}$ on days $1-3$ (PA), or cisplatin $75 \mathrm{mg} / \mathrm{m}^{2} \mathrm{IV}$ on day 1 and etoposide $100 \mathrm{mg} / \mathrm{m}^{2} \mathrm{IV}$ on day 1 , oral administration $200 \mathrm{mg} / \mathrm{m}^{2}$ on days $2-3$, or $100 \mathrm{mg} / \mathrm{m}^{2} \mathrm{IV}$ on days $1-3$ (PE). For the 88 eligible patients who started treatment, the ORR was $61 \%$ for the A arm, 77\% for the PA arm, and $63 \%$ for the PE arm. The PFS was 5.2 months for A, 6.9 for PA, and 5.8 for PE, while OS (10-11 months) was similar in all arms. The major toxicity was grade three hematological in the A, PA, and PE arms, and included neutropenia (73\%, $73 \%, 69 \%)$, thrombocytopenia $(17 \%, 15 \%, 9.4 \%)$, anemia $(10 \%, 15 \%, 3.1 \%)$ and febrile neutropenia $(13 \%, 18 \%, 6 \%)$, respectively. One treatment-related death occurred in the A arm, three in the PA arm, and three in the PE arm. This study demonstrated that the combination therapy of amrubicin with cisplatin has the highest response rate in Western patients. Further evaluation of this therapy is warranted.

Since carboplatin has less nonhematological toxicity than cisplatin, a Phase II trial of amrubicin combined with carboplatin for chemotherapy-naïve elderly patients with SCLC was carried out. ${ }^{31}$ The patients received amrubicin (35 mg/m² for 3 days) and carboplatin (AUC 4.0, day 1) every 21 days. Thirty-six patients with an average age of 76 (range 70-83) were enrolled. The ORR was $89 \%$ with one complete response and 31 partial responses. The median PFS was 5.8 months and the MST was 18.6 months. Grade 3-4 neutropenia occurred in most patients (97\%) and six (17\%) had grade 3-4 febrile neutropenia, while other toxic effects were moderate. These results demonstrated that amrubicin combined with carboplatin is effective for SCLC with acceptable toxicity even in an elderly population. To evaluate the efficacy of the combination therapy in patients with previously-treated SCLC, another Phase II trial of combination therapy of amrubicin with carboplatin was recently completed. ${ }^{39}$ Twenty-five eligible patients received amrubicin (30 mg/m²/day for 3 days) plus carboplatin (AUC $4 \mathrm{mg} \mathrm{min-}$ ute/mL using the Calvert formula on day 1) every 3 weeks. The ORR was $36.0 \%$ and the PFS was 3 months. The response rates differed significantly between patients with sensitive relapse $(58.3 \%)$ and those with refractory relapse $(15.4 \%)$. The median PFS time of patients with sensitive relapse (5 months) was significantly longer than that of patients with refractory relapse ( 2 months). The hematological toxicity was similar to previous reports, while nonhematologic toxicity was temporary and generally mild to moderately severe. To circumvent side-effects, it has been suggested that Pegfilgrastim, a long-lasting G-CSF, should be added to the regimen, and a trial with amrubicin and carboplatin with Pegfilgrastim in patients with ED-SCLC has been initiated in a population in the USA (NCT01076504).

\section{Phase III trials}

So far, only two Phase III trials of amrubicin as a single agent or in combination with cisplatin for the treatment of SCLC have been completed. The first Phase III trial of amrubicin in Western patients was reported at the American Society of Clinical Oncology annual meeting in $2011 .^{40}$ This multicenter trial was designed to compare the safety and efficacy of amrubicin versus topotecan for the second-line treatment of SCLC. A total of 637 patients were randomly assigned 2:1 to amrubicin treatment ( $40 \mathrm{mg} / \mathrm{m}^{2}$ IV on days $1-3, \mathrm{n}=424$ ) or to topotecan treatment $\left(1.5 \mathrm{mg} / \mathrm{m}^{2} \mathrm{IV}\right.$ on days $\left.1-5, \mathrm{n}=213\right)$ with prophylactic white blood cell growth factors required in the last third of the trial. Amrubicin treatment resulted in a significantly higher ORR than topotecan (31\% versus 17\%). The median PFS was 4.1 months and the median OS was 7.5 months with amrubicin, and the corresponding values were 4.0 months and 7.8 months with topotecan. The 12-month, 18-month, and 24-month OS was $28 \%, 16 \%, 9 \%$ in the amrubicin group, and $25 \%, 9 \%$, and $3 \%$ in the topotecan group, respectively. Adverse events (grade 3/4) in the amrubicin versus the topotecan groups were neutropenia ( $41 \%$ versus 53\%), thrombocytopenia $(21 \%$ versus 54\%), anemia (16\% versus 30\%), infections (16\% versus $10 \%)$, febrile neutropenia ( $10 \%$ versus $4 \%$ ), and cardiac disorders (5\% versus $5 \%){ }^{40}$ The results showed that amrubicin is more effective in the second-line treatment of SCLC with significant improvement in ORR, and with survival trending in favor of amrubicin (HR 0.88), especially in the refractory group of patients (HR 0.77).

For combination therapy with cisplatin, the early results of a Phase III trial in a Japanese population were recently reported. ${ }^{41}$ The study was designed to compare amrubicin and cisplatin (AP) with irinotecan and cisplatin (IP) for the treatment of ED-SCLC. The primary endpoint was OS and the secondary endpoints were RR, PFS adverse events, and quality of life. A total of 284 patients were randomized 1:1 to receive either IP or AP. Patients in the IP group received irinotecan (60 mg/m² IV on days 1,8 , and 15$)$, and cisplatin $\left(60 \mathrm{mg} / \mathrm{m}^{2}\right.$, IV on day 1 , every 4 weeks). Patients in the AP group received amrubicin (40 mg/m², IV on days $1-3$, and cisplatin (60 mg/ $\mathrm{m}^{2}$, IV on day 1 , every 3 weeks). One hundred ninety-one patients were enrolled, and more febrile neutropenia unexpectedly occurred in the AP group. To cope with the adverse 
events, the dose of amrubicin was then reduced to $35 \mathrm{mg} / \mathrm{m}^{2}$. The median OS of AP (15.0 months) was worse than that of IP (18.3 months). No improvement in the median PFS and ORR was found. AP showed more bone marrow suppression than expected although it caused fewer cases of diarrhea. Another multicenter Phase III trial of amrubicin with cisplatin versus etoposide-cisplatin for ED-SCLC is now being carried out in the People's Republic of China (NCT00660504), but the results have not yet been released.

These results clearly indicate that improvement of current regimens is crucial for the success of amrubicin treatment. As part of these efforts, a triplet combination regimen ${ }^{44}$ and a weekly regimen ${ }^{28}$ have been studied for the treatment of patients with SCLC and NSCLC. Very recently, an alternating regimen with amrubicin plus cisplatin and weekly administration of irinotecan plus cisplatin for ED-SCLC has been reported with good outcomes. ${ }^{43}$

\section{Future directions for amrubicin therapy}

During the last 10 years, amrubicin therapy has shown promise for the treatment of SCLC, but challenges still remain. First, finding the best regimen is the most important task. The prophylactic use of G-CSF to reduce myelosuppression and enhance the efficacy of amrubicin at a relatively low dose is a promising idea. ${ }^{44}$ Second, several lines of evidence indicate that the genetic backgrounds of patients may be very important for achieving optimal outcomes. NADPH:NQO1 and NADPH-P450 reductase are enzymes involved in the metabolism of amrubicin. ${ }^{25}$ The levels and gene polymorphisms of these enzymes in the Asian and Western population might explain the different effectiveness of the drug in patients of different ethnicities. ${ }^{45}$ Clearly, finding predictive biomarkers for the selection of patients susceptible to amrubicin treatment is the basis of successful therapy. In the future, the design of personalized regimens by single-agent or combination therapy with amrubicin may achieve ideal effects. Finally, the profound elucidation of the signaling pathways related to carcinogenesis, proliferation, apoptosis, and metastasis in SCLC is crucial for the optimization of new therapeutic regimens. It may be worthwhile to design targeted therapy with amrubicin by combining it with monoclonal antibodies or with antibody drugs to reduce the toxicity and increase efficacy and safety. ${ }^{46,47}$ Antibody-drug conjugate as a new type of targeted therapy has been successfully used in cancer-targeted therapy. ${ }^{48}$ As a rapidly expending drug modality, antibody-drug conjugates have also been actively studied for the treatment of lung cancer, including SCLC. ${ }^{48,49}$ The targeted therapy using antibody-amrubicin or amrubicinol may dramatically change therapeutic regimens and offer promise against the devastating cancer in the near future.

\section{Acknowledgments}

We thank Dr Iain Bruce, University of Hong Kong, for critical reading of the manuscript. This work was partially supported by Fundamental Research Funds for the Central Universities, People's Republic of China.

\section{Disclosure}

The authors report no conflicts of interest in this work.

\section{References}

1. Ferlay J, Shin HR, Bray F, Forman D, Mathers C, Parkin DM. Estimates of worldwide burden of cancer in 2008: GLOBOCAN 2008. Int $J$ Cancer. 2010;127(12):2893-2917.

2. Hann CL, Rudin CM. Management of small-cell lung cancer: incremental changes but hope for the future. Oncology (Williston Park, NY). 2008;22(13):1486-1492.

3. Kalemkerian GP, Akerley W, Bogner P, et al. Small cell lung cancer. J Natl Compr Canc Netw. 2013;11(1):78-98.

4. Murray N, Turrisi AT. A review of first-line treatment for small-cell lung cancer. J Thorac Oncol. 2006;1(3):270-278.

5. Govindan R, Page N, Morgensztern D, et al. Changing epidemiology of small-cell lung cancer in the United States over the last 30 years analysis of the surveillance, epidemiologic, and end results database. J Clin Oncol. 2006;24(28):4539-4544.

6. Stinchcombe TE, Gore EM. Limited-stage small cell lung cancer: current chemoradiotherapy treatment paradigms. Oncologist. 2010; 15(2):187-195.

7. Kurata T, Okamoto I, Tamura K, Fukuoka M. Amrubicin for nonsmall-cell lung cancer and small-cell lung cancer. Invest New Drugs. 2007;25(5):499-504.

8. Kimura T, Kudoh S, Hirata K. Review of the management of relapsed small-cell lung cancer with amrubicin hydrochloride. Clin Med Insights Oncol. 2011;5:23-34.

9. Shah CM. Amrubicin: a synthetic anthracyclin analogue in the treatment of extensive stage small cell lung cancer. Recent Pat Anticancer Drug Discov. 2009;4(3):241-245.

10. Hanada M, Mizuno S, Fukushima A, Saito Y, Noguchi T, Yamaoka T. A new antitumor agent amrubicin induces cell growth inhibition by stabilizing topoisomerase II-DNA complex. Jpn J Cancer Res. 1998;89(11): 1229-1238.

11. Morisada S, Yanagi Y, Noguchi T, Kashiwazaki Y, Fukui M. Antitumor activities of a novel 9-aminoanthracycline (SM-5887) against mouse experimental tumors and human tumor xenografts. Jpn J Cancer Res. 1989;80(1):69-76.

12. Noda T, Watanabe T, Kohda A, Hosokawa S, Suzuki T. Chronic effects of a novel synthetic anthracycline derivative (SM-5887) on normal heart and doxorubicin-induced cardiomyopathy in beagle dogs. Invest New Drugs. 1998;16(2):121-128.

13. Salvatorelli E, Menna P, Surapaneni S, et al. Pharmacokinetic characterization of amrubicin cardiac safety in an ex vivo human myocardial strip model. I. Amrubicin accumulates to a lower level than doxorubicin or epirubicin. J Pharmacol Exp Ther. 2012;341(2):464-473.

14. Salvatorelli E, Menna P, Gonzalez Paz O, et al. Pharmacokinetic characterization of amrubicin cardiac safety in an ex vivo human myocardial strip model. II. Amrubicin shows metabolic advantages over doxorubicin and epirubicin. J Pharmacol Exp Ther. 2012;341(2): 474-483. 
15. Yamaoka T, Hanada M, Ichii S, Morisada S, Noguchi T, Yanagi Y. Cytotoxicity of amrubicin, a novel 9-aminoanthracycline, and its active metabolite amrubicinol on human tumor cells. Jpn J Cancer Res. 1998;89(10):1067-1073.

16. Frederick CA, Williams LD, Ughetto G, et al. Structural comparison of anticancer drug-DNA complexes: adriamycin and daunomycin. Biochemistry. 1990;29(10):2538-2549.

17. Fornari FA, Randolph JK, Yalowich JC, Ritke MK, Gewirtz DA. Interference by doxorubicin with DNA unwinding in MCF-7 breast tumor cells. Mol Pharmacol. 1994;45(4):649-656.

18. Pigram WJ, Fuller W, Hamilton LD. Stereochemistry of intercalation: interaction of daunomycin with DNA. Nature New Biol. 1972;235(53): 17-19.

19. Minotti G, Menna P, Salvatorelli E, Cairo G, Gianni L. Anthracyclines: molecular advances and pharmacologic developments in antitumor activity and cardiotoxicity. Pharmacol Rev. 2004;56(2):185-229.

20. Okamoto I, Hamada A, Matsunaga Y, et al. Phase I and pharmacokinetic study of amrubicin, a synthetic 9-aminoanthracycline, in patients with refractory or relapsed lung cancer. Cancer Chemother Pharmacol. 2006;57(3):282-288.

21. Hamada A. [Development of an individualized therapy for establishing the optimal dosage by the pharmacokinetics profiles of anticancer agents.] Yakugaku Zasshi. 2005;125(8):631-637. Japanese.

22. Kimura T, Kudoh S, Mitsuoka S, et al. Plasma concentration of amrubicinol in plateau phase in patients treated for 3 days with amrubicin is correlated with hematological toxicities. Anticancer Drugs. 2009;20(6): 513-518.

23. Matsunaga Y, Hamada A, Okamoto I, et al. Pharmacokinetics of amrubicin and its active metabolite amrubicinol in lung cancer patients. Ther Drug Monit. 2006;28(1):76-82.

24. Makino Y, Yamamoto N, Sato H, et al. Pharmacokinetic and pharmacodynamic study on amrubicin and amrubicinol in Japanese patients with lung cancer. Cancer Chemother Pharmacol. 2012;69(4):861-869.

25. Tani N, Yabuki M, Komuro S, Kanamaru H. Characterization of the enzymes involved in the in vitro metabolism of amrubicin hydrochloride. Xenobiotica. 2005;35(12):1121-1133.

26. Nagata M, Kimura T, Suzumura T, et al. C609T polymorphism of NADPH quinone oxidoreductase 1 correlates clinical hematological toxicities in lung cancer patients treated with amrubicin. Clin Med Insights Oncol. 2013;7:31-39.

27. Takakuwa O, Oguri T, Ozasa H, et al. C609T polymorphism of NAD(P) $\mathrm{H}$ quinone oxidoreductase 1 as a predictive biomarker for response to amrubicin. J Thorac Oncol. 2011;6(11):1826-1832.

28. Kitagawa C, Saka H, Kajikawa S, Mori K, Oki M, Suzuki R. Phase I and pharmacologic study of weekly amrubicin in patients with refractory or relapsed lung cancer: Central Japan Lung Study Group (CJLSG) 0601 trial. Cancer Chemother Pharmacol. 2012;69(5):1379-1385.

29. Ohe Y, Negoro S, Matsui K, et al. Phase I-II study of amrubicin and cisplatin in previously untreated patients with extensive-stage small-cell lung cancer. Ann Oncol. 2005;16(3):430-436.

30. Fukuda M, Nakamura Y, Kasai T, et al; Nagasaki Thoracic Oncology Group. A phase I study of amrubicin and carboplatin for previously untreated patients with extensive-disease small cell lung cancer. JThorac Oncol. 2009;4(6):741-745.

31. Inoue A, Ishimoto O, Fukumoto S, et al. A phase II study of amrubicin combined with carboplatin for elderly patients with small-cell lung cancer: North Japan Lung Cancer Study Group Trial 0405. Ann Oncol. 2010;21(4):800-803.

32. Asakuma M, Yamamoto M, Wada M, et al. Phase I trial of irinotecan and amrubicin with granulocyte colony-stimulating factor support in extensive-stage small-cell lung cancer. Cancer Chemother Pharmacol. 2012;69(6):1529-1536.
33. Onoda S, Masuda N, Seto T, et al; Thoracic Oncology Research Group Study 0301. Phase II trial of amrubicin for treatment of refractory or relapsed small-cell lung cancer: Thoracic Oncology Research Group Study 0301. J Clin Oncol. 2006;24(34):5448-5453.

34. Inoue A, Sugawara S, Yamazaki K, et al. Randomized phase II trial comparing amrubicin with topotecan in patients with previously treated small-cell lung cancer: North Japan Lung Cancer Study Group Trial 0402. J Clin Oncol. 2008;26(33):5401-5406.

35. Kaira K, Sunaga N, Tomizawa Y, et al. A phase II study of amrubicin, a synthetic 9-aminoanthracycline, in patients with previously treated lung cancer. Lung Cancer. 2010;69(1):99-104.

36. Ettinger DS, Jotte R, Lorigan P, et al. Phase II study of amrubicin as second-line therapy in patients with platinum-refractory small-cell lung cancer. J Clin Oncol. 2010;28(15):2598-2603.

37. Jotte R, Conkling P, Reynolds C, et al. Randomized phase II trial of single-agent amrubicin or topotecan as second-line treatment in patients with small-cell lung cancer sensitive to first-line platinum-based chemotherapy. J Clin Oncol. 2011;29(3):287-293.

38. O'Brien ME, Konopa K, Lorigan P, et al. Randomised phase II study of amrubicin as single agent or in combination with cisplatin versus cisplatin etoposide as first-line treatment in patients with extensive stage small cell lung cancer - EORTC 08062. Eur J Cancer. 2011;47(15): 2322-2330.

39. Hirose T, Nakashima M, Shirai T, et al. Phase II trial of amrubicin and carboplatin in patients with sensitive or refractory relapsed small-cell lung cancer. Lung Cancer. 2011;73(3):345-350.

40. Jotte R, von Pawel J, Spigel DR, et al. Randomized phase III trial of amrubicin versus topotecan (Topo) as second-line treatment for small cell lung cancer (SCLC). In: 2011 American Society of Clinical Oncology Annual Meeting Proceedings (Post-Meeting Edition); June 3-7, 2011; Chicago, Illinois. Vol 29, No 15_suppl (May 20 Supplement), 2011:7000.

41. Kotani Y, Satouchi M, Ando A, et al. A phase III study comparing amrubicin and cisplatin (AP) with irinotecan and cisplatin (IP) for the treatment of extended-stage small cell lung cancer (ED-SCLC). In: 2012 American Society of Clinical Oncology Annual Meeting Proceedings (Post-Meeting Edition); June 1-5, 2012; Chicago, Illinois Vol 30, No 15_suppl (May 20 Supplement), 2012:7003.

42. Kobayashi M, Matsui K, Iwamoto Y, et al; West Japan Oncology Group. Phase II study of sequential triplet chemotherapy, irinotecan and cisplatin followed by amrubicin, in patients with extensive-stage small cell lung cancer: West Japan Thoracic Oncology Group Study 0301. JThorac Oncol. 2010;5(7):1075-1080.

43. Noro R, Yoshimura A, Yamamoto K, et al; East Japan Chesters Group. Alternating chemotherapy with amrubicin plus cisplatin and weekly administration of irinotecan plus cisplatin for extensive-stage small cell lung cancer. Anticancer Res. 2013;33(3):1117-1123.

44. Hata A, Katakami N, Fujita S, et al. Amrubicin at a lower-dose with routine prophylactic use of granulocyte-colony stimulating factor for relapsed small-cell lung cancer. Lung Cancer. 2011;72(2):224-228.

45. Ettinger DS. Amrubicin for the treatment of small cell lung cancer: does effectiveness cross the Pacific? J Thorac Oncol. 2007;2(2):160-165.

46. Abidin AZ, Garassino MC, Califano R, Harle A, Blackhall F. Targeted therapies in small cell lung cancer: a review. Ther Adv Med Oncol. 2010;2(1):25-37.

47. Zhan J, Han Q, Wang K. Development of antibody therapeutics for small cell lung cancer. Expert Opin Investig Drugs. 2013;22(2):235-244.

48. Scott AM, Wolchok JD, Old LJ. Antibody therapy of cancer. Nat Rev Cancer. 2012;12(4):278-287.

49. Mullard A. Maturing antibody-drug conjugate pipeline hits 30 . Nat Rev Drug Discov. 2013;12(5):329-332. 
Drug Design, Development and Therapy

Dovepress

\section{Publish your work in this journal}

Drug Design, Development and Therapy is an international, peerreviewed open-access journal that spans the spectrum of drug design and development through to clinical applications. Clinical outcomes, patient safety, and programs for the development and effective, safe, and sustained use of medicines are a feature of the journal, which

has also been accepted for indexing on PubMed Central. The manuscript management system is completely online and includes a very quick and fair peer-review system, which is all easy to use. Visit $\mathrm{http}: / / \mathrm{www}$.dovepress.com/testimonials.php to read real quotes from published authors.

Submit your manuscript here: http://www.dovepress.com/drug-design-development-and-therapy-journal 